\title{
ON THE GLOBAL FLOW OF A 3-DIMENSIONAL LOTKA-VOLTERRA SYSTEM
}

\author{
JUSTINO ALAVEZ-RAMÍREZ ${ }^{1}$, GAMALIEL BLÉ ${ }^{1}$ \\ VÍCTOR CASTELLANOS ${ }^{1}$ AND JAUME LLIBRE ${ }^{2}$
}

\begin{abstract}
In the study of the black holes with Higgs field appears in a natural way the Lotka-Volterra differential system

$$
\dot{x}=x(y-1), \quad \dot{y}=y\left(1+y-2 x^{2}-z^{2}\right), \quad \dot{z}=z y,
$$

in $\mathbb{R}^{3}$. Here we provide the qualitative analysis of the flow of this system describing the $\alpha$-limit set and the $\omega$-limit set of all orbits of this system in the whole Poincaré ball, i.e. we identify $\mathbb{R}^{3}$ with the interior of the unit ball of $\mathbb{R}^{3}$ centered at the origin and we extend analytically this flow to its boundary, i.e. to the infinity.
\end{abstract}

\section{Introduction AND STATEMENT OF THE MAIN RESUlts}

Breitenlohner et al. in their study of the black holes with Higgs field reduced the relevant terms to the following Lotka-Volterra polynomial differential system in $\mathbb{R}^{3}$ :

$$
\dot{x}=x(y-1), \quad \dot{y}=y\left(1+y-2 x^{2}-z^{2}\right), \quad \dot{z}=z y,
$$

see for more details page 441 of [4]. They, analyzing the local motion around the $z$-axis (i.e. $\mathrm{x}=\mathrm{y}=0$ ), which is formed by singular points, obtained information about the growing of the mass of a black hole with Higgs field.

The Lotka-Volterra systems are the differential systems of the form

$$
\dot{x}_{k}=x_{k} f_{k}\left(x_{1}, \ldots, x_{n}\right), \quad \text { for } k=1, \ldots, n .
$$

The name of such systems is due to the fact that Lotka and Volterra were the first in considering them in dimension 2 for studying problems coming from the ecology, see $[12,17]$. Later on Kolmogorov in [11] generalizes these systems, and then some authors called them Kolmogorov systems. There are many natural phenomena that can be modeled by the Lotka-Volterra systems such as the time evolution of conflicting species in biology [14], chemical reactions [9], plasma physics [13], hydrodynamics [5], economics [16], etc.

In this article we want to describe the $\alpha$-limit set and the $\omega$-limit set of all orbits of system (1) in $\mathbb{R}^{3}$. For a precise definition of the $\alpha$-limit set and of the $\omega$-limit set of an orbit, see for instance section 1.4 of [7].

Key words and phrases. $\alpha$-limit, $\omega$-limit, phase portrait, Lotka-Volterra system, black hole, Higgs field. 
We hope that our analysis of the $\alpha$-limit sets and of the $\omega$-limit sets of all orbits of the differential system (1) can help to the physics working with the black holes with Higgs field to go further in their results.

Since system (1) is defined in the open manifold $\mathbb{R}^{3}$, for studying its orbits in a neighborhood of infinity, we shall identify $\mathbb{R}^{3}$ with the interior of the unit ball of $\mathbb{R}^{3}$ centered at the origin, and we shall extend analytically this flow to its boundary (the infinity). This compactification is essentially due to Poincaré, this ball is called the Poincaré ball, and the extended differential system on that closed ball is called the Poincaré compatification of system (1). For precise definitions of all these notions see subsection 6.4 of the appendix. The extended flow to the Poincaré ball leaves the boundary of the ball invariant, i.e. if an orbit has a point in this boundary the whole orbit is contained in the boundary. In other words the infinity is invariant.

We shall see in section 3 that due to the symmetries of system (1) it is sufficient to study its Poincaré compactification in a quarter of the Poincaré ball, more precisely it is sufficient to the study the flow of the Poincaré compactification in the region

$$
R=\left\{(x, y, z) \in \mathbb{R}^{3}: x^{2}+y^{2}+z^{2} \leq 1, x \geq 0, z \geq 0\right\},
$$

see Figure 1.

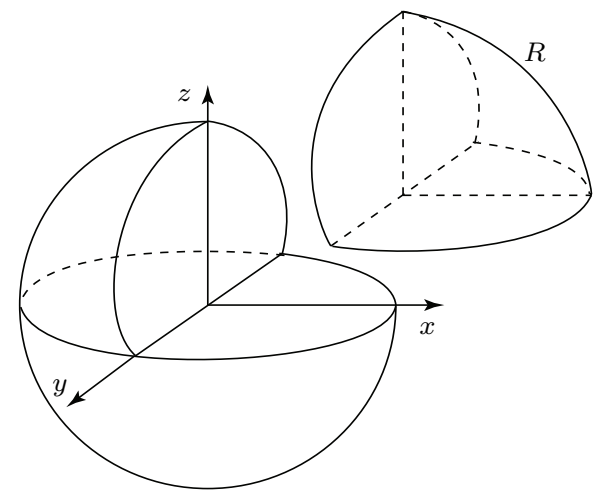

FiguRE 1. The region $R$.

Clearly the interior of $R \backslash\{y=0\}$ has two connected components:

$$
\begin{aligned}
& R_{1}=\left\{(x, y, z) \in R: x^{2}+y^{2}+z^{2}<1, x>0, y>0, z>0\right\}, \\
& R_{2}=\left\{(x, y, z) \in R: x^{2}+y^{2}+z^{2}<1, x>0, y<0, z>0\right\} .
\end{aligned}
$$

Since the planes of coordinates and the infinity are invariant by the Poincaré compactification of system (1), the four boundaries of $R_{1}$ and $R_{2}$ are invariant. As usual we denote the boundary of $R_{i}$ by $\partial R_{i}$. The flow on these 
boundaries is described in the next two theorems. For a definition of topologically equivalent phase portraits see for instance section 1.3 of [7].

Theorem 1. The following two statements hold.

(a) The phase portrait of the Poincaré compactification of system (1) on the boundaries $x=0, y=0$ and $z=0$ of $R_{1}$ is topologically equivalent to the one described in Figure 2(a).

(b) The phase portrait of the Poincaré compactification of system (1) on $R_{1}^{\infty}=\partial R_{1} \cap\left\{x^{2}+y^{2}+z^{2}=1\right\}$ (i.e. the phase portrait at the infinity of $\left.R_{1}\right)$ is topologically equivalent to the one described in Figure 2(b).

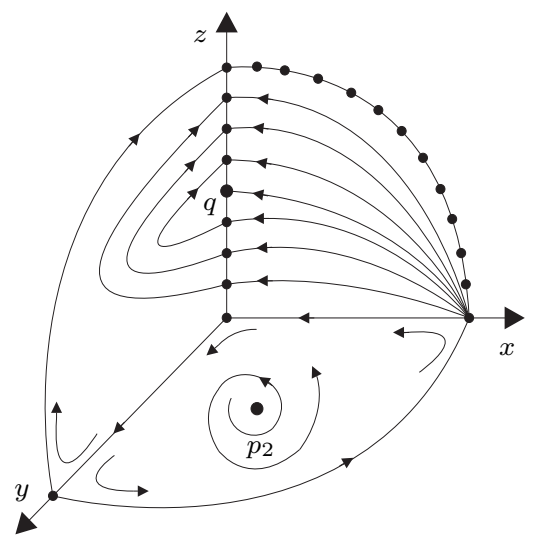

(a)

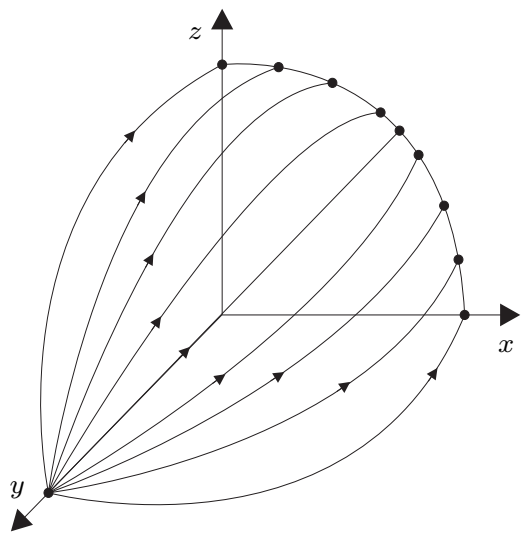

(b)

FiguRE 2. The plane portraits in the boundary of $R_{1}$.

Theorem 2. The following two statements hold.

(a) The phase portrait of the Poincaré compactification of system (1) on the boundaries $x=0, y=0$ and $z=0$ of $R_{2}$ is topologically equivalent to the one described in Figure 3(a).

(b) The phase portrait of the Poincaré compactification of system (1) on $R_{2}^{\infty}=\partial R_{2} \cap\left\{x^{2}+y^{2}+z^{2}=1\right\}$ (i.e. the phase portrait at the infinity of $\left.R_{2}\right)$ is topologically equivalent to the one described in Figure 3(b).

Theorems 1 and 2 are proved in section 4 .

We say that a $C^{1}$ function $H(t, x, y, z)$ is an invariant of the differential system (1) if $H(t, x, y, z)=$ constant, for all values of $t$ for which the solution $(x(t), y(t), z(t))$ is defined. When an invariant function is independent of the time, then it is called a first integral.

Proposition 3. System (1) in the open region $R_{1} \cup R_{2}$ has the invariant $H(t, x, y, z)=e^{t} x / z$. 


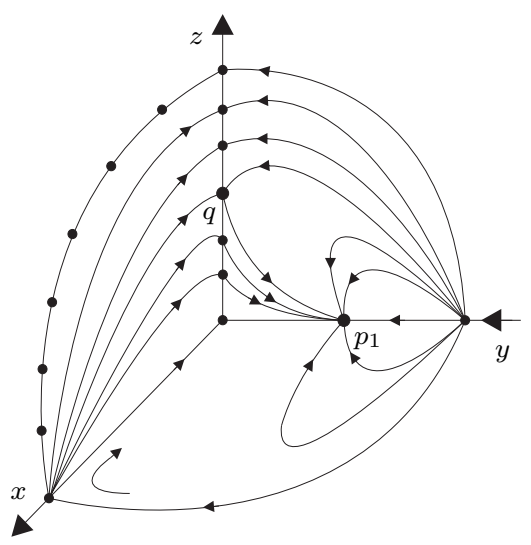

(a)

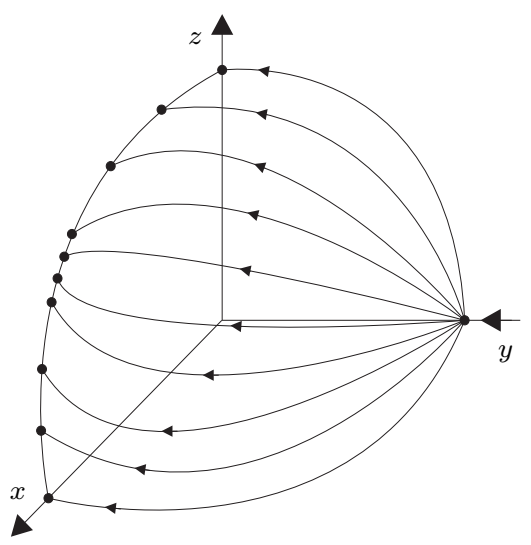

(b)

FiguRE 3. The plane portraits in the boundary of $R_{2}$.

Proposition 3 is proved in section 5 .

As we shall see in section 2 system (1) has three isolated singular points in $\mathbb{R}^{3}$, namely

$$
P_{1}=(0,-1,0), \quad P_{2}=(1,1,0), \quad P_{3}=(-1,1,0) .
$$

Moreover $P_{1}$ is a local attractor, and $P_{2}$ and $P_{3}$ are local repeller. Let $P$ be the diffeomorphism such that $P\left(\mathbb{R}^{3}\right)$ is equal to the interior of the Poincaré ball. Then we denote the three isolated finite singular points in the Poincaré ball by $p_{i}=P\left(P_{i}\right)$ for $i=1,2,3$.

In the next two theorems we describe the $\alpha$-and the $\omega$-limit sets of all the orbits contained in $R_{1} \cup R_{2}$. Let $\sigma_{z}$ be the segment of the $z$-axis contained in $R$ having with endpoints the points $q=P((0,0,1))$ and $(0,0,1)$, closed in $q$ and open in $(0,0,1)$, see Figure $2($ a) or Figure $3(\mathrm{a})$. We define

$$
\sigma_{\infty}=\left\{(x, 0, z) \in R: x^{2}+z^{2}=1\right\} .
$$

So $\sigma_{\infty}$ are together with the two points $(0, \pm 1,0)$ are the singular points at the infinity of $R$, see Figures 2(b) and 3(b).

Theorem 4. Let $\gamma$ be an orbit of system (1) such that $P(\gamma) \subset R_{1}$.

(a) The $\alpha$-limit set of $P(\gamma)$ is either the finite singular point $p_{2}$, or an infinite singular point of $\sigma_{\infty} \cup\{(0,1,0)\}$.

(b) The $\omega$-limit set of $P(\gamma)$ is either some of the finite singular points of $\sigma_{z}$, or some of the infinity singular points $\sigma_{\infty} \cup\{(0,1,0)\}$.

Theorem 5. Let $\gamma$ be an orbit of system (1) such that $P(\gamma) \subset R_{2}$.

(a) The $\alpha$-limit set of $P(\gamma)$ is an infinite singular point of $\sigma_{\infty} \cup\{(0,-1,0)\}$.

(b) The $\omega$-limit set of $P(\gamma)$ is either some of the finite singular points of $\left\{p_{1}\right\} \cup \sigma_{z}$, or some of the infinity singular points $\sigma_{\infty} \cup\{(0,-1,0)\}$. 
Theorems 4 and 5 are proved in section 5 .

We remark that all the orbits of the Poincaré compactification of system (1) contained in $R_{1} \cup R_{2}$ are heteroclinic or homoclinic because all their $\alpha^{-}$ and $\omega$-limit sets are a singular point.

Looking at the proofs of Theorems 4 and 5 , if follows that if we can study the local phase portraits of the Poincaré compactfication of system (1) at the infinite singular points (i.e. at the points $\sigma_{\infty} \cup\{(0, \pm 1,0)\}$ ), then the results of those theorems can be improved in the sense that we can show that some of the possible $\alpha$ - and $\omega$-limit sets described in Theorems 4 and 5 cannot occur. We note that two of the eigenvalues at the infinite singular points of $\sigma_{\infty}$ are zero, and that the three eigenvalues at the singular points $\{(0, \pm 1,0)\}$ are zero. So these singular points are strongly degenerate.

\section{Finite Singular points}

First we shall study the finite singular points of system (1). That is, the solutions of the system

$$
x(y-1)=0, \quad y\left(y+1-2 x^{2}-z^{2}\right)=0, \quad z y=0 .
$$

Clearly the $z$-axis is formed by singular points, and additionally there are the three isolated singular points $P_{i}$ for $i=1,2,3$ described in (3).

Now we analyze the local phase portrait at these three isolated singular points. The eigenvalues of the linear part at these singular points are

$$
-1,-1 \text { and }-2 \text { for } p_{1}, \quad \frac{1 \pm i \sqrt{15}}{2} \text { and } 1 \text { for } p_{2} \text { and } p_{3} \text {. }
$$

Hence these three singular points are hyperbolic, see subsection 6.2. Therefore $p_{1}$ is a local attractor, and $p_{2}$ and $p_{3}$ are local repeller.

\section{Symmetries}

One of the characteristic facts of the Lotka-Volterra systems in $\mathbb{R}^{3}$ is that the three coordinates planes are invariant by the flow of these systems, i.e. if an orbit of the system has a point on one of such planes that the full orbit is contained in it. Consequently the three axes are also invariant by the flow.

The differential system (1) has two symmetries.

(i) System (1) is invariant under the symmetry $(x, y, z) \rightarrow(-x, y,-z)$, i.e. the flow of it is symmetric with respect to the $y$-axis. Therefore, since the $y$-axis is invariant, if $(x(t), y(t), z(t))$ is a solution of (1) which does not intersect the $y$-axis, then $(-x(t), y(t),-z(t))$ is another solution of (1).

(ii) System (1) is invariant under the symmetry $(x, y, z) \rightarrow(-x, y, z)$, i.e. the flow of it is symmetric with respect to the plane $x=0$. So, since the plane $x=0$ is invariant, if $(x(t), y(t), z(t))$ with $x(t) \neq 0$ is a solution of $(1)$, then $(-x(t), y(t), z(t))$ is another solution of $(1)$. 
Using these two symmetries it is sufficient to describe the flow of system (1) only in a quarter of the Poincaré ball. Thus, we shall describe this flow only in the region $R$ defined in (2).

\section{Phase portraits on the invariant Planes AND AT INFinity}

In this section we first describe the phase portrait of system (1) in the intersection of the three planes of coordinates with the region $R$, and after on the boundary of $R$ satisfying that $x^{2}+y^{2}+z^{2}=1$, i.e. on the infinity.

4.1. Phase portrait on $R \cap\{z=0\}$. The flow restricted to the invariant plane $z=0$ is given by the differential system

$$
\dot{x}=x(y-1)=P(x, y), \quad \dot{y}=y\left(1+y-2 x^{2}\right)=Q(x, y) .
$$

Of course the $x$-axis and the $y$-axis are invariant. Since we are interested on the phase portrait on $R \cap\{z=0\}$, we only consider the phase portrait of system (4) in the half-plane $x \geq 0$.

In the half-plane $x \geq 0$ system (4) has exactly the three finite singular points:

$$
p_{0}=(0,0), \quad p_{1}=(0,-1), \quad p_{2}=(1,1) .
$$

The eigenvalues of the linear part at these singular points are

$$
-1,1 \text { for } p_{0}, \quad-2,-1 \text { for } p_{1}, \quad \frac{1 \pm i \sqrt{15}}{2} \text { for } p_{2} .
$$

Therefore, from subsection 6.1 it follows that $p_{0}$ is a saddle, $p_{1}$ is an attractor node, and $p_{2}$ is an unstable focus.

Now we shall study the infinity of system (4). Thus this system in the local chart $U_{1}$ becomes

$$
\dot{z}_{1}=-2 z_{1}+2 z_{1} z_{2}^{2}, \quad \dot{z}_{2}=-z_{1} z_{2}^{2}+z_{2}^{3},
$$

see subsection 6.3 for more details. Clearly the unique infinity singular point of this system is the $(0,0)$, i.e. the unique singular point on $z_{2}=0$. Using the results of subsection 6.1 , the singular point $(0,0)$ is a semi-hyperbolic saddle.

Now it only remains to study if the point $(0,0)$ of the local chart $U_{2}$ is a singular point. System (4) in the local chart $U_{2}$ writes

$$
\dot{z}_{1}=2 z_{1}^{3}-2 z_{1} z_{2}^{2}, \quad \dot{z}_{2}=-z_{2}^{2}+2 z_{1}^{2} z_{2}-z_{2}^{3} .
$$

So the $(0,0)$ is a linearly zero singular point, using the notation introduced in subsection 6.1. In order to describe its local phase portrait we shall use the blow-up technique, see the end of subsection 6.1. Then we do the change of variables $\left(z_{1}, z_{2}\right) \rightarrow\left(z_{1}, u\right)$ where $z_{2}=u z_{1}$. In the new variables system (5) becomes

$$
\dot{z}_{1}=2 z_{1}^{3}-2 z_{1}^{3} u^{2}, \quad \dot{u}=-z_{1} u^{2}+z_{1}^{2} u^{3} .
$$


Rescaling the independent variable or time by $z_{1}$, system (6) goes over to

$$
\dot{z}_{1}=2 z_{1}^{2}-2 z_{1}^{2} u^{2}, \quad \dot{u}=-u^{2}+z_{1} u^{3} .
$$

Now doing the blow-up $\left(z_{1}, u\right) \rightarrow\left(z_{1}, v\right)$ where $u=v z_{1}$ system (7) writes

$$
\dot{z}_{1}=2 z_{1}^{2}-2 z_{1}^{4} v^{2}, \quad \dot{v}=-2 z_{1} v-z_{1} v^{2}+z_{1}^{3} v^{3} .
$$

Rescaling the independent variable by $z_{1}$, system (8) becomes

$$
\dot{z}_{1}=2 z_{1}-2 z_{1}^{3} v^{2}, \quad \dot{v}=-2 v-v^{2}+z_{1}^{2} v^{3} .
$$

System (9) has the two singular points $(0,0)$ and $(0,-2)$ on the $v$-axis, having their linear part eigenvalues $-2,2$ and 2,2 respectively. Therefore the local phase portrait of system (9) around the $v$-axis is given in Figure $4(9)$. Then the local phase portrait of system (8) around the $v$-axis is given in Figure 4(8). Going back through the blow-up $u=v z_{1}$ the local phase portrait of system (7) around the $u$-axis is given in Figure 4(7). So the local phase portrait of system (6) around the $v$-axis is given in Figure 4(6). Again going back through the blow-up $z_{2}=u z_{1}$ the local phase portrait of system (5) around the $z_{2}$-axis is given in Figure 4(5).

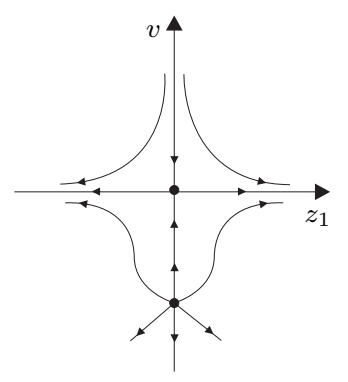

(9)

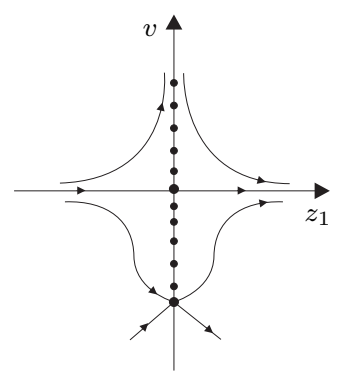

(8)

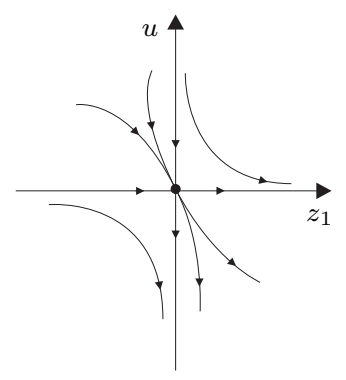

(7)

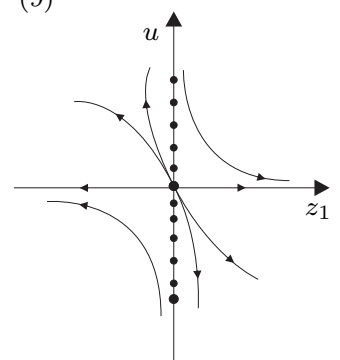

(6)

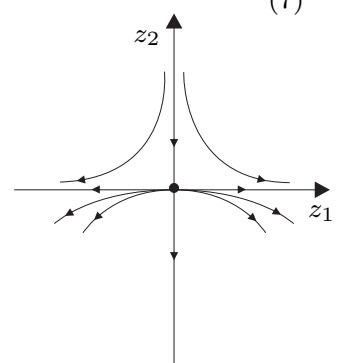

(5)

Figure 4. The blow-up development of the singular point $(0,0)$ of $U_{2}$.

Now we know the local phase portraits at all finite and infinite singular points of system (4) in the half-plane $x \geq 0$. Recalling that the $x$-axis is invariant, that a periodic orbit must surround at least a singular point (this 
follows from the Poincaré-Bendixson Theorem) and using the PoincaréBendixson Theorem (see for instance Corollary 1.30 of [7]), we get that the phase portrait of system (4) in the half-plane $x \geq 0$ of the Poincaré disc is homeomorphic to the one given in Figure 2(a) and 3(a) restricted to $z=0$, except that perhaps there can be periodic orbits surrounding the focus $p_{2}$.

Let $B=B(x, y)=1 /(x y)$. Since system (4) in the simply connected region $x>0$ satisfies

$$
\frac{\partial(B P)}{\partial x}+\frac{\partial(B Q)}{\partial y}=\frac{1}{x},
$$

by the Dulac's criterion (see Theorem 7.12 of [7]) it follows that system (4) has no periodic orbits in $x>0$. Hence the phase portrait of system (4) in the half-plane $x \geq 0$ of the Poincaré disc is homeomorphic to the one given in Figure 2(a) and 3(a) restricted to $z=0$.

4.2. Phase portrait on $R \cap\{x=0\}$. The flow restricted to the invariant plane $x=0$ is given by the differential system

$$
\dot{y}=y\left(1+y-z^{2}\right), \quad \dot{z}=z y .
$$

Of course the $z$-axis is formed by singular points. We remove it rescaling the independent variable by $y$, then we get the system

$$
\dot{y}=1+y-z^{2}, \quad \dot{z}=z .
$$

Since we are interested on the phase portrait on $R \cap\{x=0\}$, we only consider the phase portrait of system (11) in the half-plane $z \geq 0$.

The unique finite singular point of system (11) is $p_{1}=(-1,0)$. The eigenvalues of the linear part of system (11) at $p_{1}$ are 1 and 1 . So $p_{1}$ is an unstable node.

Now we study the infinite singular points of system (11). Thus in the local chart $U_{1}$ it writes

$$
\dot{z}_{1}=z_{1}^{3}-z_{1} z_{2}^{2}, \quad \dot{z}_{2}=-z_{2}^{2}+z_{1}^{2} z_{2}-z_{2}^{3} .
$$

This system has a unique singular point at infinity, the $(0,0)$ which is linearly zero. Note that system (12) is very similar to system (5). In fact we can study the local phase portrait of system (12) doing the same blow-ups that we did for analyzing system (5), and we shall obtain the same local phase portrait, i.e. the local phase portrait given in Figure 4(5).

System (11) in the local chart $U_{2}$ becomes

$$
\dot{z}_{1}=-1+z_{2}^{2}, \quad \dot{z}_{2}=-z_{2}^{2} .
$$

Since the $(0,0)$ of $U_{2}$ is not a singular point, the unique singular points of system (11) at infinity are the endpoints of the $y$-axis.

We know the local phase portraits at all finite and infinite singular points of system (11) in the half-plane $z \geq 0$. Recalling that the $y$-axis is invariant, by the Poincaré-Bendixson Theorem, we obtain the phase portrait of system (11) in the half-plane $z \geq 0$ of the Poincaré disc. Going back through the 
rescaling of time the phase portrait of system (11) in the half-plane $z \geq 0$, we get the phase portrait of system (10) in the half-plane $z \geq 0$ of the Poincaré disc given in Figures 2(a) and 3(a) restricted to $x=0$.

Looking at Figures 2(a) and 3(a) we observe two different local behaviors at the singular points of the positive $z$-axis contained in $R \cap\{x=0\}$, and one bifurcation singular point (i.e. one singular point in which the local phase portrait changes). The eigenvalues of the linear part of system (10) at the singular point $\left(0, z_{0}\right)$ with $z_{0}>0$ are 0 and $1-z_{0}^{2}$. Hence the bifurcation singular point is $(0,1)$. We denote by $q$ the point in $R$ (i.e. after the Poincaré compactification) corresponding to the point $(0,1)$ of system (10).

4.3. Phase portrait at the infinity of $R$. Since we know the behavior of the flow at the infinity of $R \cap\{x=0\}$, for studying the infinity of $R$ it is sufficient to analyze the flow at the infinity of the local chart $U_{1}$, see subsection 6.4. In this local chart system (1) writes

$$
\dot{z}_{1}=-2 z_{1}-z_{1} z_{2}^{2}+2 z_{1} z_{3}^{2}, \quad \dot{z}_{2}=z_{2} z_{3}^{2}, \quad \dot{z}_{3}=-z_{1} z_{3}^{2}+z_{3}^{3} .
$$

System (13) restricted at the infinity (i.e. at $z_{3}=0$ ) becomes

$$
\dot{z}_{1}=-2 z_{1}-z_{1} z_{2}^{2}, \quad \dot{z}_{2}=0 .
$$

So the unique singular points at infinity of the local chart $U_{1}$ are all the points of axis $z_{1}=z_{3}=0$. Since system (14) has the straight lines $z_{2}=$ constant invariant by the flow, $\dot{z}_{1}<0$ if $z_{1}>0$ and $\dot{z}_{1}>0$ if $z_{1}<0$, the phase portrait of system (1) at the infinity of $R$ is described in Figures 2(b) and $3(\mathrm{~b})$.

4.4. Phase portrait on $R \cap\{y=0\}$. The flow restricted to the invariant plane $y=0$ is given by the differential system

$$
\dot{x}=-x, \quad \dot{z}=0 .
$$

So the straight lines $z=$ constant are formed by orbits of the system, and all the points of the $z$-axis are singular. The infinity of $R \cap\{y=0\}$ follows from subsection 4.3. So the phase portrait on the invariant set $R \cap\{y=0\}$ is given in the Figure 2(a) and 3(a) restricted to $y=0$.

Note that putting together the results of the previous four subsections we obtain the proofs of Theorems 1 and 2 .

$$
\text { 5. The } \alpha \text { - And } \omega \text {-Limit Sets of the orbits in } R_{1} \cup R_{2}
$$

In this section we study the flow in the open regions $R_{1}$ and $R_{2}$.

Proof of Proposition 3. Since $H=H(t, x, y)=e^{t} x / z$, it is immediate to check that

$$
\frac{d H}{d t}=\frac{\partial H}{\partial x} \dot{x}+\frac{\partial H}{\partial y} \dot{y}+\frac{\partial H}{\partial z} \dot{z}+\frac{\partial H}{\partial t}=0,
$$

where $(\dot{x}, \dot{y}, \dot{z})$ are given in (1). Therefore $H$ is an invariant of system (1). This completes the proof of the proposition. 
Now we shall describe how we get the invariant $H$. The planes $x=$ $0, y=0$ and $z=0$ are invariant algebraic surfaces (see section 8.4 of [7] for a definition). We remark that in [7] we define invariant algebraic curves instead of invariant algebraic surfaces but the definition extends in the natural way to higher dimension, as well as the Darboux theory of integrability. Then, using the notations and definitions of Chapter 8 of [7], the invariant planes $x=0, y=0$ and $z=0$ have cofactors $k_{1}=y-1$, $k_{2}=y+1-2 x^{2}-z^{2}$ and $k_{3}=y$, respectively. Since the solution of the equation

$$
\lambda_{1} k_{1}+\lambda_{2} k_{2}+\lambda_{3} k_{3}+1=0
$$

is $\lambda_{1}=1, \lambda_{2}=0$ and $\lambda_{3}=-1$, from Theorem 8.7 of [7], we get that

$$
H(t, x, y)=e^{t} x^{\lambda_{1}} y^{\lambda_{2}} z^{\lambda_{3}}=e^{t} x / z,
$$

is an invariant of system (1).

Let $\gamma=\{(x(t), y(t), z(t)): t \in(a, b)\}$ be an orbit of system (1) in the open region $\mathcal{R}_{1}=\left\{(x, y, z) \in \mathbb{R}^{3}: x>0, y>0, z>0\right\}$ being the open interval $(a, b)$ its maximal interval of definition such that $-\infty \leq a<0<b \leq+\infty$. Recall that $P$ denotes the diffeomorphism such that $P\left(\mathbb{R}^{3}\right)$ is equal to the interior of the Poincaré ball. Then $P\left(\mathcal{R}_{1}\right)=R_{1}$.

It is well known that if $-\infty<a$ then the $\alpha$-limit set of $P(\gamma)$ is contained in $R_{1}^{\infty}$, and if $b<+\infty$ then the $\omega$-limit set of $P(\gamma)$ is also contained $R_{1}^{\infty}$. For more details see Theorem 1.2 of [7], this theorem in [7] is proved for 2dimensional differential systems but the proof for higher dimensions is the same. Similar results hold if $\gamma$ is an orbit of system (1) in the open region $R_{2}$.

We say that the orbit $\gamma \subset R_{i}$ with $\mathrm{i}=1,2$ is positively bounded if there exists a neighborhood $N$ of $R_{i}^{\infty}$ in $R$ such that $P(\{(x(t), y(t), z(t)): t \in$ $(0, b)\}) \cap N=\emptyset$. So, in particular $b=+\infty$. Similarly we say that the orbit $\gamma$ is negatively bounded if there exists a neighborhood $N$ of $R_{i}^{\infty}$ in $R$ such that $P(\{(x(t), y(t), z(t)): t \in(a, 0)\}) \cap N=\emptyset$. So, in particular $a=-\infty$.

Lemma 6. Let $\gamma=\{(x(t), y(t), z(t)): t \in(a, b)\}$ be an orbit of system (1) such that $P(\gamma) \subset R_{i}$ for $i=1,2$ being the open interval $(a, b)$ its maximal interval of definition such that $-\infty \leq a<0<b \leq+\infty$.

(a) If $-\infty<a$, then the $\alpha$-limit set of $P(\gamma) \subset R_{i}^{\infty}$.

(b) If $b<+\infty$, then the $\omega$-limit set of $P(\gamma) \subset R_{i}^{\infty}$.

(c) If $\gamma$ is negatively bounded, then $a=-\infty$ and $\lim _{t \rightarrow-\infty} z(t)=0$.

(d) If $\gamma$ is positively bounded, then $b=+\infty$ and $\lim _{t \rightarrow+\infty} x(t)=0$.

Proof. Statements (a) and (b) follows directly from the paragraphs just before the statement of the lemma.

A negatively bounded orbit always has $-\infty=a$, as we have seen. From the definition of negatively bounded orbit in $R_{i}$, it follows that there exists 
$M>0$ such that $0<x(t)<M$ and $0<z(t)<M$ for all $t<0$. Then, by Proposition $3, H(t, x(t), y(t), z(t))=h$ with $h>0$. So

$$
\frac{z(t)}{x(t)}=\frac{e^{t}}{h} \quad \text { for all } t<0 .
$$

Now taking limit in (15) when $t \rightarrow-\infty$, we obtain that

$$
\lim _{t \rightarrow-\infty} \frac{z(t)}{x(t)}=0 .
$$

Therefore, since the orbit $(x(t), y(t), z(t))$ is negatively bounded, we get that

$$
\lim _{t \rightarrow-\infty} z(t)=0 .
$$

So statement (c) is proved.

A positively bounded orbit always has $b=+\infty$, as we have seen before. From the definition of positively bounded orbit in $R_{i}$, it follows that there exists $M>0$ such that $0<x(t)<M$ and $0<z(t)<M$ for all $t>0$. Then, by Proposition $3, H(t, x(t), y(t), z(t))=h$ with $h>0$. So

$$
\frac{z(t)}{x(t)}=\frac{e^{t}}{h} \quad \text { for all } t>0 .
$$

Taking limit in (16) when $t \rightarrow+\infty$, we obtain that

$$
\lim _{t \rightarrow+\infty} \frac{z(t)}{x(t)}=+\infty .
$$

Therefore, since the orbit $(x(t), y(t), z(t))$ is positively bounded, we get that

$$
\lim _{t \rightarrow+\infty} x(t)=0 .
$$

Hence statement $(\mathrm{d})$ is proved.

The following result already appeared in page 441 of [4].

Lemma 7. The singular points $(0,0, z)$ with $z \geq 0$ and $z \neq 1$ of system (1) are normally hyperbolic. Moreover for every of such points there is a local invariant surface through it such that the local phase portrait at the singular point $(0,0, z)$ is a saddle if $0 \leq z<1$, and a stable node if $z>1$.

Proof. The eigenvalues at the singular point $(0,0, z)$ are $0,-1$ and $1-z^{2}$. So, from the theory of the normal hyperbolic singular points (see [10]) the lemma follows.

Proof of Theorem 4. Let $\gamma=\{(x(t), y(t), z(t)): t \in(a, b)\}$ be an orbit of system (1) such that $P(\gamma) \subset R_{1}$ being the open interval $(a, b)$ its maximal interval of definition such that $-\infty \leq a<0<b \leq+\infty$. First we study the $\alpha$-limit set of $P(\gamma)$. We distinguish three cases.

Case 1: Assume that the orbit $\gamma$ is negatively bounded. Then, by Lemma $6(\mathrm{c})$, we have that $\lim _{t \rightarrow-\infty} z(t)=0$. So the points of its $\alpha$-limit set must be finite (because $\gamma$ is negatively bounded) and their $z$ coordinate is zero. 
Consequently, from Theorem 1(a), it follows that the $\alpha$-limit set of $P(\gamma)$ only can be either the singular point $p_{2}$, or the singular point $(0,0,0)$. But, Lemma 7 and the Figures 2(a) say that the singular point $(0,0,0)$ cannot be the $\alpha$-limit set of an orbit leaving in $R_{1}$.

Case 2: Suppose that $-\infty<a$. Therefore, by Lemma 6(a) and Figure 2(b), we have that the $\alpha$-limit set of $P(\gamma)$ must be an infinite singular point of $\sigma_{\infty} \cup\{(0,1,0)\}$.

Case 3: Finally, assume that $\gamma$ is non negatively bounded and $-\infty=a$. Therefore, $P(\gamma)$ when $t \rightarrow-\infty$ is as close as we want to $R_{1}^{\infty}$, and from the theorem of continuous dependence of the solutions of an ordinary differential equation respect to the initial conditions and from Figure 2(b), it follows that the $\alpha$-limit set of $P(\gamma)$ must be an infinite singular point of $\sigma_{\infty} \cup\{(0,1,0)\}$. In short statement (a) of Theorem 4 is proved.

Now we study the $\omega$-limit set of $P(\gamma)$. Again we distinguish three cases.

Case 1: Assume that the orbit $\gamma$ is positively bounded. Then, by Lemma $6(\mathrm{~d})$, we have that $\lim _{t \rightarrow+\infty} x(t)=0$. So the points of its $\omega$-limit set must be finite (because $\gamma$ is positively bounded) and their $x$ coordinate is zero. Consequently, from the Figure 2(a), it follows that the $\omega$-limit set of $P(\gamma)$ only can be the finite singular points of the $z$-axis. Now, by Lemma 7 , only the points $\sigma_{z}$ of the $z$-axis can be the $\alpha$-limit sets of the orbits leaving in $R_{1}$.

Case 2: Suppose that $b<+\infty$. Therefore, by Lemma 6(b) and Theorem 1 (b), we have that the $\omega$-limit set of $P(\gamma)$ must be an infinite singular point of $\sigma_{\infty} \cup\{(0,1,0)\}$.

Case 3: Finally, assume that $\gamma$ is non positively bounded and $b=+\infty$. Therefore $P(\gamma)$ when $t \rightarrow+\infty$ is as close as we want to $R_{1}^{\infty}$, and from the theorem of continuous dependence of the solutions of an ordinary differential equation respect to the initial conditions and from Figure 2(b), it follows that the $\omega$-limit set of $P(\gamma)$ must be an infinite singular point of $\sigma_{\infty} \cup\{(0,1,0)\}$. In short statement (b) of Theorem 4 is proved.

Proof of Theorem 5. Let $\gamma=\{(x(t), y(t), z(t)): t \in(a, b)\}$ be an orbit of system (1) such that $P(\gamma) \subset R_{2}$ being the open interval $(a, b)$ its maximal interval of definition such that $-\infty \leq a<0<b \leq+\infty$. First we study the $\alpha$-limit set of $P(\gamma)$. We distinguish three cases.

Case 1: Assume that the orbit $\gamma$ is negatively bounded. Then, by Lemma $6(\mathrm{c})$, we have that $\lim _{t \rightarrow-\infty} z(t)=0$. So the points of its $\alpha$-limit set must be finite (because $\gamma$ is negatively bounded) and their $z$ coordinate is zero. Consequently, from the Figure 3(a), it follows that the $\alpha$-limit set of $P(\gamma)$ only can be the singular point $(0,0,0)$. But by Lemma 7 the singular point $(0,0,0)$ cannot be the $\alpha$-limit of an orbit contained in $R_{2}$. Hence there are no orbits in $R_{2}$ which are negatively bounded. 
Case 2: Suppose that $-\infty<a$. Therefore, by Lemma 6(a) and Figure 3(b), we have that the $\alpha$-limit set of $P(\gamma)$ must be an infinite singular point of $\sigma_{\infty} \cup\{(0,-1,0)\}$.

Case 3: Finally, assume that $\gamma$ is non negatively bounded and $-\infty=a$. Therefore $P(\gamma)$ when $t \rightarrow-\infty$ is as close as we want to $R_{2}^{\infty}$, and from the theorem of continuous dependence of the solutions of an ordinary differential equation respect to the initial conditions and from Figure 3(b), it follows that the $\alpha$-limit set of $P(\gamma)$ must be an infinite singular point of $\sigma_{\infty} \cup\{(0,-1,0)\}$. In short statement (a) of Theorem 5 is proved.

Now we study the $\omega$-limit set of $P(\gamma)$. Again we distinguish three cases.

Case 1: Assume that the orbit $\gamma$ is positively bounded. Then, by Lemma $6(\mathrm{~d})$, we have that $\lim _{t \rightarrow+\infty} x(t)=0$. So the points of its $\omega$-limit set must be finite (because $\gamma$ is positively bounded) and their $x$ coordinate is zero. Consequently, from the Figure 3(a) and Lemma 7, it follows that the $\omega$-limit set of $P(\gamma)$ only can some of the singular points of $\left\{p_{1}\right\} \cup \sigma_{z}$.

Case 2: Suppose that $b<+\infty$. Therefore, by Lemma 6(b) and Figure 3(b), we have that the $\omega$-limit set of $P(\gamma)$ must be an infinite singular point of $\sigma_{\infty} \cup\{(0,-1,0)\}$.

Case 3: Finally, assume that $\gamma$ is non positively bounded and $b=+\infty$. Therefore $P(\gamma)$ when $t \rightarrow+\infty$ is as close as we want to $R_{2}^{\infty}$, and from the theorem of continuous dependence of the solutions of an ordinary differential equation respect to the initial conditions and from Figure $3(\mathrm{~b})$, it follows that the $\omega$-limit set of $P(\gamma)$ must be an infinite singular point of $\sigma_{\infty} \cup\{(0,-1,0)\}$. In short statement (b) of Theorem 4 is proved.

\section{THE APPENDIX}

In this appendix we summarize the basic results from the qualitative theory of ordinary differential equations that we need for studying the global phase portrait of the Lotka-Volterra differential system (1).

6.1. Singular points of $\mathbb{R}^{2}$. Now we shall describe briefly the tools that we need for studying the local phase portrait at a singular point in $\mathbb{R}^{2}$. While we have tools for studying the local phase portraits of every isolated singular point in dimension 2 (see for instance [7]), such a tool do not exist in dimension 3 .

A point $p \in \mathbb{R}^{2}$ is said to be a singular point of the vector field $X=(P, Q)$ if $P(p)=Q(p)=0$. We recall first some results which hold when $P$ and $Q$ are analytic functions in a neighborhood of $p$. As usual $P_{x}$ denotes the partial derivative of $P$ with respect to the variable $x$.

If $\Delta=P_{x}(p) Q_{y}(p)-P_{y}(p) Q_{x}(p)$ and $T=P_{x}(p)+Q_{y}(p)$, then the singular point $p$ is said to be non-degenerate if $\Delta \neq 0$. Then $p$ is an isolated singular point. Moreover, $p$ is a saddle if $\Delta<0$, a node if $T^{2} \geq 4 \Delta>0$ (stable if $T<0$, unstable if $T>0$ ), a focus if $4 \Delta>T^{2}>0$ (stable if $T<0$, unstable 
if $T>0$ ), and either a weak focus or a center if $T=0<\Delta$; for more details see [2], p. 183, or Theorem 2.15 of [7].

A singular point $p$ is called hyperbolic if the two eigenvalues of the Jacobian matrix $D X(p)$ have nonzero real part. So, the hyperbolic singular points are the non-degenerate ones except the weak focus and the centers.

A degenerate singular point $p$ (i.e. $\Delta=0$ ) with $T \neq 0$ is called semihyperbolic, and $p$ is isolated in the set of all singular points. Now we summarize the results on semi-hyperbolic singular points that we shall need in this paper, for a proof see Theorem 65 of [2], or Theorem 2.19 of [7].

Theorem 8. Let $(0,0)$ be an isolated point of the vector field $(F(x, y), y+$ $G(x, y)$ ), where $F$ and $G$ are analytic functions in a neighborhood of the origin starting at least with quadratic terms in the variables $x$ and $y$. Let $y=g(x)$ be the solution of the equation $y+G(x, y)=0$ in a neighborhood of $(0,0)$. Assume that the development of the function $f(x)=F(x, g(x))$ is of the form $f(x)=\mu x^{m}+$ HOT (Higher Order Terms), where $m \geq 2$ and $\mu \neq 0$. When $m$ is odd, then $(0,0)$ is either an unstable node, or a saddle depending if $\mu>0$, or $\mu<0$, respectively. In the case of the saddle the stable separatrices are tangent to the $x$-axis. If $m$ is even, then $(0,0)$ is a saddle-node, i.e. the singular point is formed by the union of two hyperbolic sectors with one parabolic sector. The stable separatrix is tangent to the positive (respectively negative) $x$-axis at $(0,0)$ according to $\mu<0$ (respectively $\mu>0$ ). The two unstable separatrices are tangent to the $y$-axis at $(0,0)$.

The singular points which are non-degenerate or semi-hyperbolic are called elementary.

When $\Delta=T=0$ but the Jacobian matrix at $p$ is not the zero matrix and $p$ is isolated in the set of all singular points, we say that $p$ is nilpotent. The local phase portrait of these points has been studied in [1], or Theorems 66 and 67 and the simplified scheme of Section 22.3 of [2], or Theorem 3.5 of [7]. But since they do not appear in the analysis of our system we do not describe these results here.

Finally, if the Jacobian matrix at the singular point $p$ is identically zero, and $p$ is isolated inside the set of all singular points, then we say that $p$ is linearly zero. The study of its local phase portrait needs a special treatment (directional blow-ups), see for more details [3], or Chapter 3 of [7].

6.2. Hyperbolic singular points of $\mathbb{R}^{n}$. A singular point $p$ of the differential system $\dot{x}=X(x)$ in $\mathbb{R}^{n}$ is called hyperbolic if all the eigenvalues of the Jacobian matrix $D X(p)$ have nonzero real part. The following result reduces the study of the local phase portrait at a hyperbolic singular point to study the phase portrait of its linear part, for a proof see for instance [15]. 
Theorem 9 (Grobman-Hartman). The local phase portrait in a sufficiently small neighborhood of a hyperbolic singular point $p$ of a $C^{1}$ differential system in $\mathbb{R}^{n}$ is homeomorphic to the phase portrait of the linear part of the differential system at $p$.

6.3. Poincaré compactification of $\mathbb{R}^{2}$. Let $X=(P, Q)$ be a planar polynomial vector field of degree $n$. The Poincaré compactified vector field $p(X)$ corresponding to $X$ is an analytic vector field induced on $\mathbb{S}^{2}$ as follows (see, for instance [8], or Chapter 5 of [7]). Let $\mathbb{S}^{2}=\left\{y=\left(y_{1}, y_{2}, y_{3}\right) \in \mathbb{R}^{3}\right.$ : $\left.y_{1}^{2}+y_{2}^{2}+y_{3}^{2}=1\right\}$ (the Poincaré sphere) and $T_{y} \mathbb{S}^{2}$ be the tangent space to $\mathbb{S}^{2}$ at point $y$. Consider the central projection $f: T_{(0,0,1)} \mathbb{S}^{2} \rightarrow \mathbb{S}^{2}$. This map defines two copies of $X$, one in the northern hemisphere and the other in the southern hemisphere. Denote by $X^{\prime}$ the vector field $D f \circ X$ defined on $\mathbb{S}^{2}$ except on its equator $\mathbb{S}^{1}=\left\{y \in \mathbb{S}^{2}: y_{3}=0\right\}$. Clearly $\mathbb{S}^{1}$ is identified to the infinity of $\mathbb{R}^{2}$. In order to extend $X^{\prime}$ to a vector field on $\mathbb{S}^{2}$ (including $\mathbb{S}^{1}$ ) it is necessary that $X$ satisfies suitable conditions. In the case that $X \in \mathcal{P}_{n}\left(\mathbb{R}^{2}\right)$, $p(X)$ is the only analytic extension of $y_{3}^{n-1} X^{\prime}$ to $\mathbb{S}^{2}$. On $\mathbb{S}^{2} \backslash \mathbb{S}^{1}$ there are two symmetric copies of $X$, and knowing the behavior of $p(X)$ around $\mathbb{S}^{1}$, we know the behavior of $X$ at infinity. The projection of the closed northern hemisphere of $\mathbb{S}^{2}$ on $y_{3}=0$ under $\left(y_{1}, y_{2}, y_{3}\right) \longmapsto\left(y_{1}, y_{2}\right)$ is called the Poincaré disc, and it is denoted by $\mathbb{D}^{2}$. The Poincaré compactification has the property that $\mathbb{S}^{1}$ is invariant under the flow of $p(X)$.

In this paper we say that two polynomial vector fields $X$ and $Y$ on $\mathbb{R}^{2}$ are topologically equivalent if there exists a homeomorphism on $\mathbb{S}^{2}$ preserving the infinity $\mathbb{S}^{1}$ carrying orbits of the flow induced by $p(X)$ into orbits of the flow induced by $p(Y)$, preserving or reversing simultaneously the sense of all orbits.

As $\mathbb{S}^{2}$ is a differentiable manifold, for computing the expression for $p(X)$, we can consider the six local charts $U_{i}=\left\{y \in \mathbb{S}^{2}: y_{i}>0\right\}$, and $V_{i}=\{y \in$ $\left.\mathbb{S}^{2}: y_{i}<0\right\}$ where $i=1,2,3$; and the diffeomorphisms $F_{i}: U_{i} \rightarrow \mathbb{R}^{2}$ and $G_{i}: V_{i} \rightarrow \mathbb{R}^{2}$ for $i=1,2,3$ are the inverses of the central projections from the planes tangent at the points $(1,0,0),(-1,0,0),(0,1,0),(0,-1,0),(0,0,1)$ and $(0,0,-1)$ respectively. If we denote by $z=\left(z_{1}, z_{2}\right)$ the value of $F_{i}(y)$ or $G_{i}(y)$ for any $i=1,2,3$ (so $z$ represents different things according to the local charts under consideration), then some easy computations give for $p(X)$ the following expressions:

$$
\begin{aligned}
& \text { (17) } z_{2}^{n} \Delta(z)\left(Q\left(\frac{1}{z_{2}}, \frac{z_{1}}{z_{2}}\right)-z_{1} P\left(\frac{1}{z_{2}}, \frac{z_{1}}{z_{2}}\right),-z_{2} P\left(\frac{1}{z_{2}}, \frac{z_{1}}{z_{2}}\right)\right) \text { in } U_{1} \text {, } \\
& \text { (18) } z_{2}^{n} \Delta(z)\left(P\left(\frac{z_{1}}{z_{2}}, \frac{1}{z_{2}}\right)-z_{1} Q\left(\frac{z_{1}}{z_{2}}, \frac{1}{z_{2}}\right),-z_{2} Q\left(\frac{z_{1}}{z_{2}}, \frac{1}{z_{2}}\right)\right) \text { in } U_{2} \text {, } \\
& \Delta(z)\left(P\left(z_{1}, z_{2}\right), Q\left(z_{1}, z_{2}\right)\right) \quad \text { in } \quad U_{3} \text {, }
\end{aligned}
$$

where $\Delta(z)=\left(z_{1}^{2}+z_{2}^{2}+1\right)^{-\frac{1}{2}(n-1)}$. The expression for $V_{i}$ is the same as that for $U_{i}$ except for a multiplicative factor $(-1)^{n-1}$. In these coordinates for $i=1,2, z_{2}=0$ always denotes the points of $\mathbb{S}^{1}$. We omit the factor $\Delta(z)$ 
by rescaling the vector field $p(X)$. Thus we obtain a polynomial vector field in each local chart.

6.4. Poincaré compactification of $\mathbb{R}^{3}$. In $\mathbb{R}^{3}$ we consider the polynomial differential system

$$
\dot{x}=P^{1}(x, y, z), \quad \dot{y}=P^{2}(x, y, z), \quad \dot{z}=P^{3}(x, y, z),
$$

or equivalently its associated polynomial vector field $X=\left(P^{1}, P^{2}, P^{3}\right)$. The degree $n$ of $X$ is defined as $n=\max \left\{\operatorname{deg}\left(P^{i}\right): i=1,2,3\right\}$.

Let $\Sigma^{3}=\left\{y=\left(y_{1}, y_{2}, y_{3}, y_{4}\right) \in \mathbb{R}^{4}:\|y\|=1\right\}$ be the unit sphere in $\mathbb{R}^{4}$, and

$$
\Sigma_{+}=\left\{y \in \Sigma^{3}: y_{4}>0\right\} \quad \text { and } \quad \Sigma_{-}=\left\{y \in \Sigma^{3}: y_{4}<0\right\}
$$

be the northern and southern hemispheres, respectively. The tangent space to $\Sigma^{3}$ at the point $y$ is denoted by $T_{y} \Sigma^{3}$. Then, the tangent hyperplane

$$
T_{(0,0,0,1)} \Sigma^{3}=\left\{\left(x_{1}, x_{2}, x_{3}, 1\right) \in \mathbb{R}^{4}:\left(x_{1}, x_{2}, x_{3}\right) \in \mathbb{R}^{3}\right\}
$$

is identified with $\mathbb{R}^{3}$.

We consider the central projections

$$
f_{+}: \mathbb{R}^{3}=T_{(0,0,0,1)} \Sigma^{3} \rightarrow \mathbb{S}_{+} \quad \text { and } \quad f_{-}: \mathbb{R}^{3}=T_{(0,0,0,1)} \Sigma^{3} \rightarrow \mathbb{S}_{-},
$$

defined by

$$
f_{+}(x)=\frac{1}{\Delta x}\left(x_{1}, x_{2}, x_{3}, 1\right) \quad \text { and } \quad f_{-}(x)=-\frac{1}{\Delta x}\left(x_{1}, x_{2}, x_{3}, 1\right),
$$

where $\Delta x=\left(1+\sum_{i=1}^{3} x_{i}^{2}\right)^{1 / 2}$. Through these central projections, $\mathbb{R}^{3}$ can be identified with the northern and the southern hemispheres, respectively. The equator of $\Sigma^{3}$ is $\Sigma^{2}=\left\{y \in \Sigma^{3}: y_{4}=0\right\}$. Clearly, $\Sigma^{2}$ can be identified with the infinity of $\mathbb{R}^{3}$.

The maps $f_{+}$and $f_{-}$define two copies of $X$, one $D f_{+} \circ X$ in the northern hemisphere and the other $D f_{-} \circ X$ in the southern one. Denote by $\bar{X}$ the vector field on $\Sigma^{3} \backslash \Sigma^{2}=\Sigma_{+} \cup \Sigma_{-}$which restricted to $\Sigma_{+}$coincides with $D f_{+} \circ X$ and restricted to $\Sigma_{-}$coincides with $D f_{-} \circ X$.

In what follows we shall work with the orthogonal projection of the closed northern hemisphere to $y_{4}=0$. Note that this projection is a closed ball $B$ of radius one, whose interior is diffeomorphic to $\mathbb{R}^{3}$ and whose boundary $\Sigma^{2}$ corresponds to the infinity of $\mathbb{R}^{3}$. We shall extend analytically the polynomial vector field $\bar{X}$ to the boundary, in such a way that the flow on the boundary is invariant. This new vector field on $B$ will be called the Poincaré compactification of $X$, and $B$ will be called the Poincaré ball. Poincaré introduced this compactification for polynomial vector fields in $\mathbb{R}^{2}$, and its extension to $\mathbb{R}^{m}$ can be found in [6]. 
The expression for $\bar{X}(y)$ on $\Sigma_{+} \cup \Sigma_{-}$is

$$
\bar{X}(y)=y_{4}\left(\begin{array}{ccc}
1-y_{1}^{2} & -y_{2} y_{1} & -y_{3} y_{1} \\
-y_{1} y_{2} & 1-y_{2}^{2} & -y_{3} y_{2} \\
-y_{1} y_{3} & -y_{2} y_{3} & 1-y_{3}^{2} \\
-y_{1} y_{4} & -y_{2} y_{4} & -y_{3} y_{4}
\end{array}\right)\left(\begin{array}{l}
P^{1} \\
P^{2} \\
P^{3}
\end{array}\right),
$$

where $P^{i}=P^{i}\left(y_{1} /\left|y_{4}\right|, y_{2} /\left|y_{4}\right|, y_{3} /\left|y_{4}\right|\right)$. Written in this way $\bar{X}(y)$ is a vector field in $\mathbb{R}^{4}$ tangent to the sphere $\Sigma^{3}$.

Now we can extend analytically the vector field $\bar{X}(y)$ to the whole sphere $\Sigma^{3}$ by

$$
p(X)(y)=y_{4}^{n-1} \bar{X}(y)
$$

this extended vector field $p(X)$ is called the Poincaré compactification of $X$.

As $\Sigma^{3}$ is a differentiable manifold, to compute the expression for $p(X)$ we can consider the eight local charts $\left(U_{i}, F_{i}\right),\left(V_{i}, G_{i}\right)$ where $U_{i}=\{y \in$ $\left.\Sigma^{3}: y_{i}>0\right\}$, and $V_{i}=\left\{y \in \Sigma^{3}: y_{i}<0\right\}$ for $i=1,2,3,4$; the diffeomorphisms $F_{i}: U_{i} \rightarrow \mathbb{R}^{3}$ and $G_{i}: V_{i} \rightarrow \mathbb{R}^{3}$ for $i=1,2,3,4$ are the inverses of the central projections from the origin to the tangent planes at the points $( \pm 1,0,0,0),(0, \pm 1,0,0),(0,0, \pm 1,0)$ and $(0,0,0, \pm 1)$, respectively. We now do the computations on $U_{1}$. Suppose that the origin $(0,0,0,0)$, the point $\left(y_{1}, y_{2}, y_{3}, y_{4}\right) \in \Sigma^{3}$ and the point $\left(1, z_{1}, z_{2}, z_{3}\right)$ in the tangent plane to $\Sigma^{3}$ at $(1,0,0,0)$ are collinear, then we have

$$
\frac{1}{y_{1}}=\frac{z_{1}}{y_{2}}=\frac{z_{2}}{y_{3}}=\frac{z_{3}}{y_{4}}
$$

and consequently

$$
F_{1}(y)=\left(\frac{y_{2}}{y_{1}}, \frac{y_{3}}{y_{1}}, \frac{y_{4}}{y_{1}}\right)=\left(z_{1}, z_{2}, z_{3}\right)
$$

defines the coordinates on $U_{1}$.

As

$$
D F_{1}(y)=\left(\begin{array}{cccc}
-y_{2} / y_{1}^{2} & 1 / y_{1} & 0 & 0 \\
-y_{3} / y_{1}^{2} & 0 & 1 / y_{1} & 0 \\
-y_{4} / y_{1}^{2} & 0 & 0 & 1 / y_{1}
\end{array}\right)
$$

and $y_{4}^{n-1}=\left(\frac{z_{3}}{\Delta z}\right)^{n-1}$, the analytical field $p(X)$ in $U_{1}$ becomes

$$
\frac{z_{3}^{n}}{(\Delta z)^{n-1}}\left(-z_{1} P^{1}+P^{2},-z_{2} P^{1}+P^{3},-z_{3} P^{1}\right),
$$

where $P^{i}=P^{i}\left(1 / z_{3}, z_{1} / z_{3}, z_{2} / z_{3}\right)$.

In a similar way we can deduce the expressions of $p(X)$ in $U_{2}$ and $U_{3}$. These are

$$
\frac{z_{3}^{n}}{(\Delta z)^{n-1}}\left(-z_{1} P^{2}+P^{1},-z_{2} P^{2}+P^{3},-z_{3} P^{2}\right),
$$


where $P^{i}=P^{i}\left(z_{1} / z_{3}, 1 / z_{3}, z_{2} / z_{3}\right)$ in $U_{2}$, and

$$
\frac{z_{3}^{n}}{(\Delta z)^{n-1}}\left(-z_{1} P^{3}+P^{1},-z_{2} P^{3}+P^{2},-z_{3} P^{3}\right)
$$

where $P^{i}=P^{i}\left(z_{1} / z_{3}, z_{2} / z_{3}, 1 / z_{3}\right)$ in $U_{3}$.

The expression for $p(X)$ in $U_{4}$ is $z_{3}^{n+1}\left(P^{1}, P^{2}, P^{3}\right)$ where the component $P^{i}=P^{i}\left(z_{1}, z_{2}, z_{3}\right)$. The expression for $p(X)$ in the local chart $V_{i}$ is the same as in $U_{i}$ multiplied by $(-1)^{n-1}$.

When we shall work with the expression of the compactified vector field $p(X)$ in the local charts we omit the factor $1 /(\Delta z)^{n-1}$. We can do that through a rescaling of the time.

We remark that all the points on the sphere at infinity in the coordinates of any local chart have $z_{3}=0$.

\section{ACKNOWLEDGEMENTS}

The first author is partially supported by the grant PROMEP/103.5/08/3189. The first three authors are partially supported by two CONACYT grants with numbers 58968 and 62613 . The fourth author has been partially supported by the grants MCYT/FEDER MTM2005-06098-C02-01 and CIRIT 2005SGR 00550 .

\section{REFERENCES}

[1] A. F. AndreEv, Investigation of the behaviour of the integral curves of a system of two differential equations in the neighborhood of a singular point, Translation of Amer. Math. Soc. 8 (1958), 183-207.

[2] A. A. Andronov, E. A. Leontovich, I. I. Gordon, And A. L. Maier, Qualitative Theory of Second-Order Dynamical Systems, Wiley, New York, 1973.

[3] V. I. Arnold and Y. S. Ilyashenko, Dynamical Systems I, Ordinary Differential Equations. Encyclopaedia of Mathematical Sciences, Vols 1-2, Springer-Verlag, Heidelberg, 1988.

[4] P. Breitenlohner, G. Lavrelashvili and D. Maison, Mass inflation and chaotic behaviour inside hairy black holes, Nuclear Physics B 524 (1998), 427-443.

[5] F.H. Busse, Transition to turbulence via the statistical limit cycle route, in Synergetics, Springer-Verlag, Berlin, 1978, p. 39.

[6] A. Cima And J. Llibre, Bounded polynomial vector fields, Trans. Amer. Math. Soc. 318 (1990), 557-579.

[7] F. Dumortier, J. Llibre And J.C. Artés, Qualitative theory of planar differential systems, UniversiText, Springer-Verlag, New York, 2006.

[8] E.A. GonzÁlez, Generic properties of polynomial vector fields at infinity, Trans. Amer. Math. Soc. 143 (1969), 201-222.

[9] R.H. HeRInG, Oscillations in Lotka-Volterra systems of chemical reactions, J. Math. Chemistry 5 (1990), 197-202.

[10] M.W. Hirsch, C.C. Pugh And M. Shub, Invariant manifolds, Lecture Notes in Mathematics, Vol. 583, Springer-Verlag, Berlin-New York, 1977.

[11] A. Kolmogorov, Sulla teoria di Volterra della lotta per l'esistenza, Giornale dell' Istituto Italiano degli Attuari 7 (1936), 74-80.

[12] A.J. LотKA, Analytical note on certain rhythmic relations in organic systems, Proc. Natl. Acad. Sci. U.S. 6 (1920), 410-415. 
[13] G. Laval and R. Pellat, Plasma Physics, Proceedings of Summer School of Theoretical Physics, Gordon and Breach, New York, 1975.

[14] R.M. MaY, Stability and Complexity in Model Ecosystems, Princeton, New Jersey, 1974.

[15] J. Palis And W. De Melo, Geometric theory of dynamical systems: an introduction, Springer-Verlag, 1982.

[16] S. Solomon And P. Richmond, Stable power laws in variable economies; LotkaVolterra implies Pareto-Zipf, The European Phsysical J. B 27 (2002), 257-261.

[17] V. VolterRa, Lecons sur la Théorie Mathématique de la Lutte pour la vie, GauthierVillars, Paris, 1931.

1 División Académica de Ciencias Básicas, UJat, Km 1 Carretera CunduacÁn-Jalpa de Méndez, C.P. 86690 Cunduacán TABasco, México

E-mail address: justino.alavez@dacb.ujat.mx, gble@ujat.mx, vicas@ujat.mx

2 Departament de Matemàtiques, Universitat Autònoma de Barcelona, 08193 Bellaterra, Barcelona, Catalonia, Spain

E-mail address: jllibre@mat.uab.cat 